\title{
Struktur politischer Öffentlichkeiten auf Twitter am Beispiel österreichischer Innenpolitik
}

\author{
Stephan Schlögl ${ }^{1,{ }^{*}}$, Axel Maireder ${ }^{1}$ \\ 1 Global Social Media Intelligence Center der GfK, Wien, Austria \\ * E-Mail: stephan.schloegl@univie.ac.at
}

\section{Zusammenfassung}

Der Beitrag nimmt die Struktur der österreichischen politischen Twittersphäre in einer Clusteranalyse der Accountverknüpfungen zwischen den NutzerInnen in den Blick. Diese zeigt I3 größere Communities, die als (Teil-)Öffentlichkeiten im Sinne themenspezifischer verdichteter Kommunikationsräume verstanden werden können. Auf Basis netzwerkanalytischer Merkmale und einer qualitativen Analyse der Nutzerbeschreibungen können acht politisch kategorisierbare und zwei regional verwurzelte Öffentlichkeiten identifiziert werden, die an der Peripherie des Netzwerks positioniert sind. Im Zentrum stehen dagegen ein Medien-Cluster und eine Community mit zivilgesellschaftlichen Akteuren, die beide Vermittlungsfunktionen für die politischen Cluster einnehmen. Die Ergebnisse dieser Analyse werden vor dem Hintergrund der österreichischen Parteienlandschaft diskutiert und mit Blick auf die Frage nach der Veränderung politischer Kommunikationsprozesse durch Soziale Onlinenetzwerke interpretiert.

\section{Schlüsselwörter}

Öffentlichkeit, Teilöffentlichkeit, Netzwerkanalyse, Twitter, Communities, Österreich

\section{Public spheres and Twitter: the case of Austrian domestic politics}

\section{Abstract}

This paper focuses on the structure of the Austrian Political Twitter sphere by studying the follower relations between very active users. We find 13 communities, which we understand as ,publics' in the sense of thematically distinguishable spheres of communication. Based on network characteristics and qualtitive analyses of profiles, we identify eight clusters with more-or-less clear political tendencies and two clusters with nodes that share common localities, all of them in the periphery of the network. A media cluster and a community of civil society actors are at the network's center, mediating between the other clusters. We interpret our findings in relation to Austria's political constellation and discuss them with regard to the changes of political communication due to the increasing importance of social media.

\section{Keywords}

Public Sphere, publics, Network Analysis, Twitter, Community, Austria

\section{Danksagung}

Die Forschung, auf der der vorliegende Aufsatz basiert, wurde im Rahmen des Projektes „Towards an Analytics of Networked Publics" in Kooperation mit dem GfK Social Media Intelligence Center (Wien) und finanziert vom GfK Verein (Nürnberg) durchgeführt.

The authors have declared that no competing interests exist. 


\section{Einleitung}

Der Einzug der neu gegründeten liberalen Partei NEOS ins Parlament war in den Augen vieler politischer Beobachter die Überraschung der österreichischen Nationalratswahlen 20I3. Mangels bekannter Persönlichkeiten und mit anderen Parteien vergleichbarer finanzieller Mittel setzten NEOS von Anfang an stark auf Soziale Onlinenetzwerke, um die Partei, die KandidatInnen und das politische Programm bekannt zu machen, und sich im innenpolitischen Diskurs zu positionieren. Aber auch andere wahlwerbende Parteien und Kandidaten setzten im vergangenen Wahlkampf Facebook, Twitter und Youtube professionell und mehr-oder-weniger erfolgreich ein. So prägten Tweets, Facebook-Posts und Youtube-Videos den innenpolitischen Diskurs intensiv mit. Insbesondere Twitter wurde dabei als wichtiger Kanal zur Diskussion aktueller Themen und zur Diffusion von Nachrichten weiter etabliert, wobei die Relevanz des Dienstes für den öffentlichen Diskurs weniger der Breite der Nutzung als der gesellschaftlichen Position der beteiligten Akteure geschuldet war: PolitikerInnen, ExpertInnen, AktivistInnen und nicht zuletzt JournalistInnen nahmen an den vielfach sehr lebendig geführten Konversationen teil, wobei letztere in ihren Medien vielfach über Twitter-Konversationen berichteten und diese so in den massenmedialen Diskurs einbrachten.

Der vorliegende Aufsatz nimmt die Twitter-Kommunikation im Vorfeld der Nationalratswahl 2013 in den Blick und fragt nach jenen grundlegenden sozio-technischen Strukturen, die der Nutzung von Twitter als Medium für den innenpolitischen Diskurs zu Grunde liegen: die über die Praxis des gegenseitigen „Folgens" hergestellten Accountnetzwerke. Diese Netzwerke, so argumentieren wir im Folgenden, sind einerseits Ausdruck spezifischer Interessenskonstellationen, und prägen anderseits die Praxis der Beteiligung auf der Mikro- und die Dynamiken der Kommunikation auf der Makroebene des politischen Diskurses wesentlich mit. Durch die in diesem Aufsatz vorgestellte Analyse von Netzwerkstrukturen und ihrer Interpretation vor dem Hintergrund der Politik- und Medienlandschaft des Landes lassen sich einerseits Erkenntnisse zur Relevanz bestimmter Akteure und Akteursgruppen für die konkreten innenpolitischen Diskussionen, als auch zur allgemeineren Frage nach der Veränderung politischer Kommunikationsprozesse durch Soziale Onlinenetzwerke gewinnen. Mit der Analyse von Accountnetzwerken rund um ein spezifisches Themenfeld gehen wir zudem einen neuen methodischen Weg in der Erforschung von Netzöffentlichkeit(en). Dabei verstehen wir die Strukturen kommunikativer Artefakte (digitaler Objekte und ihrer Verknüpfungen) als Ausdruck gesellschaftlicher Kommunikationsstrukturen, die diese Strukturen gleichzeitig mitprägen. Die theoretische und methodologische Begründung unseres Vorgehens (Abschnitt 3) sowie die Beschreibung des Forschungsdesigns (4) folgt auf einen kur- zen Überblick zu Twitter und Politik sowie Twitter in Österreich (2). Nach der Präsentation der Ergebnisse (5) und ihrer Interpretation (6) schließt der Aufsatz mit zusammenfassenden Betrachtungen vor dem Hintergrund der österreichischen Parteien- und Medienlandschaft (7).

\section{Forschungsstand}

\subsection{Twitter und politische Kommunikation}

Die Struktur des Internets als potenziell hierarchiefreies und nicht-lineares Kommunikationssystem hat seit den I990er-Jahren Erwartungen an die verstärkte Inklusion sozialer Akteure in das politische System geweckt (Anduiza et al. 2009; Farrell 20I2), die sich in den letzten Jahren rund um den Web 2.O.-Diskurs wieder verstärkt haben. Gerade Twitter erscheint auf Grund seines hochgradig offenen Zugangs und der verteilten Kommunikationsstruktur den Erwartungen an ein „demokratisches“ Medium besonders nahe zu kommen. Entsprechend vielfältig ist auch die Forschung zu Twitter und Politik, wobei sich ein großer Teil der bisher zum Thema veröffentlichten Aufsätze mit der Twitter-Nutzungspraxis von PolitikerInnen - insbesondere ihrer Interaktionsorientierung - auseinandersetzt. Dabei zeigt sich vielfach, dass die Möglichkeiten zur niederschwelligen und direkten Kommunikation mit potenziellen WählerInnen oftmals nicht wahrgenommen werden. Stattdessen dominieren traditionelle Kampagnenpraktiken: Tweets werden vielfach im Stil von Pressemeldungen veröffentlicht, garniert mit Alltagsanekdoten und persönlichen Fotos (Graham et al. 20I3; Grant et al. 20IO; Goldbeck et al., 20IO; Larsson/Moe 2OII).

Studien, die politische Kommunikation in einem weiteren Sinne verstehen (Henn et al. 20I3) und politische Akteure aus Journalismus und Zivilgesellschaft in ihre Analysen mitaufnehmen, zeigen dagegen, dass Twitter durchaus intensiv zur politischen Diskussion genutzt wird (Ausserhofer/Maireder 20I3; Bruns/ Burgess 20IIb; Bruns/Highfield 20I3; Jürgens/Jungherr 20II; Maireder/Schlögl 20I4). In Netzwerkanalysen auf Basis der Interaktionen zwischen Accounts (@-mentions) erscheinen vereinzelt auch politische AmtsträgerInnen zentral - wobei Bruns und Highfield (20I3) darauf hinweisen, dass viele NutzerInnen dazu tendieren, sich stärker über PolitikerInnen und deren Twitterauftritte auszutauschen als mit ihnen. In allen Studien zeigt sich eine starke Konzentration auf einzelne Akteure, die mit Jürgens/Jungherr (2OII) als „neue Gatekeeper“ bezeichnet werden können. Ausserhofer und Maireder (2013) zeigen, dass es sich hierbei neben bekannten Persönlichkeiten oftmals 
auch um NutzerInnen handelt, die keine herausragenden Rollen im politischen System einnehmen. Dies sind beispielsweise junge JournalistInnen, parlamentarische HinterbänklerInnen, LokalpolitikerInnen, politische AktivistInnen oder Politik-ExpertInnen. Die Analysen legen auch nahe, dass sich politische Kommunikationsprozesse auf Twitter durch die Inklusion sozialer Akteure von außerhalb des zentralen politischen Systems auszeichnen, wie dies Davis (2009) für Internetkommunikation im Allgemeinen feststellt.

So haben sich auf Twitter spezifische Kommunikationsräume entwickelt, in denen sich Individuen in ihren Rollen als (im weitesten Sinne) politische Akteure (aus Politik, Journalismus und Zivilgesellschaft) über aktuelle politische Ereignisse und Entwicklungen austauschen. Von zentraler Bedeutung für den Zugang in dieses Netzwerk sind die jeweiligen Kontakte (Followees) der NutzerInnen, die ihre individuellen Beobachtungsfelder, die „Newsfeeds“, strukturieren.Während @mention-Netzwerke Relevanzzuschreibungen in und durch Interaktionen repräsentieren, zeigen Followernetzwerke Relevanz- und Interessensstrukturen für Twitters Informationsfunktion und liegen den individuellen Beobachtungs- und Handlungsfeldern für die Nutzung des Dienstes zu Grunde. Die im vorliegenden Aufsatz vorgestellte Studie nimmt diese Strukturen für eine spezifische Akteursgruppe in Österreichs innenpolitischer „Twittersphäre“ in den Blick.

\subsection{Twitter und Politik in Österreich}

Der Beginn der wissenschaftlichen und breiteren öffentlichen Auseinandersetzung mit Twitter und Politik in Österreich geht auf die „Unibrennt“-Proteste im Herbst 2009 zurück. Von den Studierenden, die den größten Hörsaal der Universität Wien für über zwei Monate besetzt hielten, wurde Twitter als zentraler Kanal zur Mobilisierung von UnterstützerInnen und Koordination von Protestmaßnahmen genutzt. Gleichzeitig wurde auf Twitter intensiv über die Besetzung diskutiert, sowohl innerhalb der Protestgruppe als auch mit Kritikern der Proteste und JournalistInnen(Edelmann et al. 20II; Herwig et al. 20IO; Maireder/Schwarzenegger 2012, Neumayer et al. 20II).

Zur öffentlichen Wahrnehmung von Twitter als Medium politischer Kommunikation in Österreich hat in den Jahren danach vor allem der Fernsehjournalist Armin Wolf beigetragen, der seit 2010 nicht nur regelmäßig twittert, sondern zu Twitter als journalistisches Medium auch vielfach prominent interviewt wurde. ${ }^{\mathrm{I}}$

I http://www.thegap.at/rubriken/stories/artikel/im-zentrum-vontwitter-politik-und-medien/; http://www.format.at/articles/ı226/ 585/332199/armin-wolf-ich-lady-gaga.; http://kurier.at/menschen/ im-gespraech/armin-wolf-im-interview-ueber-twitter-mein-gottdann-weiss-man-halt-dass-ich-mad-men-mag/I3.728.4I2
20IO wurde die Zahl der österreichischen Twitter-NutzerInnen auf nur 20.000 geschätzt, ${ }^{2}$ wobei sich damals wie heute besonders viele JournalistInnen am Informationsaustausch auf Twitter beteiligen und auch vielfach journalistische Inhalte vermittelt werden (Maireder 2010). Armin Wolf war dabei lange Zeit der Nutzer mit den meisten Followern ${ }^{3}$ und führt auch heute noch viele einschlägige Twitter Charts an. ${ }^{4}$ Mit der Zeit wurden zunehmend auch PolitikerInnen und andere Akteure des politischen Systems auf Twitter aktiv, die sich untereinander, als auch mit BürgerInnen (also NutzerInnen ohne spezifische politische Funktion in Österreich), zu unterschiedlichen tagespolitischen Themen intensiv austauschen und so eine spezifische innenpolitische "Twittersphäre" schaffen, wie Ausserhofer/Maireder (2013) zeigen.

Die vorliegende Untersuchung schließt an diese Arbeit lose inhaltlich an, wobei zwischen den Untersuchungsperioden der beiden Studien 18 Monate liegen. In diesem Zeitraum hat sich die Zahl der aktiven TwitternutzerInnen in Österreich von geschätzten 50.000 auf rund $100.000^{5}$ verdoppelt und zahlreiche zusätzliche politische Akteure haben begonnen Twitter zu nutzen. Der Untersuchungszeitraum im ersten Halbjahr 2013 fällt zudem auf eine innenpolitisch ereignisreiche Zeit, die vom Vorwahlkampf für die Nationalratswahlen im September, dem Auftreten neuer politische Parteien (Team Stronach, NEOS), einer Volksabstimmung und vier Landtagswahlen geprägt war.

\section{Forschungsperspektive}

\section{1 Öffentlichkeit(en)}

Unsere Perspektive auf Twitterkommunikation ist öffentlichkeitstheoretisch, wobei wir nicht normativ sondern strukturtheoretisch argumentieren. Wir verstehen Öffentlichkeit als hoch komplexes Netzwerk, das sich in unzählige geographisch, kulturell, thematisch oder funktional differenzierbare Kommunikationssphären verzweigt, die vielfach miteinander verknüpft und ineinander verschränkt sind (Habermas I998). Diese Sphären können als Räume verdichteter Kommunikation verstanden werden, die sich „über solche Verdichtungsprozesse sozialräumlich voneinander unterscheiden lassen" (Hepp et al. 2012, 22). Solche Verdichtungen kommen insbesondere dann zu Stande, wenn soziale Akteure zu geteilten Problemen und Interessen kommu-

2 http://www.digirati.eu/bigideas/2010/o4/oI/twitter-reichweite-inosterreich

3 http://digitalaffairs.at/2010/03/31/twitter-in-oesterreich-wer-hatdie-meisten-follower/

4 http://www.socialmediaradar.at/twitter_ranking; http://twitterlist. ots.at/journalistinnen-und-journalisten/

$5 \mathrm{http}: / /$ socialmediaradar.at/ 
nizieren und dabei aufeinander Bezug nehmen. Wenn wir hier und im Folgenden alsovon Öffentlichkeiten oder einer spezifischen Öffentlichkeit schreiben, meinen wir nicht Öffentlichkeit als theoretisches Gesamtkonstrukt, sondern konkrete Kommunikationssphären im Sinne von „Teilöffentlichkeiten“ („publics“). Die Akteure in solchen Öffentlichkeiten sind im Hinblick auf das Interesse an einem bestimmten Thema oder Problem homogen (Gruning/Hunt 1984). Dabei können Öffentlichkeiten Kommunikationszusammenhänge umfassen, an denen jeweils ganz unterschiedlich viele Menschen beteiligt sind, die also jeweils spezifische Größen oder Reichweiten aufweisen (Emirbayer/Sheller 1999). Die vielschichtige Verknüpfung der vielfältigen Öffentlichkeiten zeigt sich unter anderem darin, dass Themen in unterschiedlichen Sphären verhandelt werden und von einer Sphäre zur nächsten wandern können (Benkler 2007; Anderson 20IO).

Jedes Individuum ist in unterschiedlichste kleine und größere Öffentlichkeiten eingebunden und fungiert als Schnittstelle, indem es Informationen zwischen unterschiedlichen Kommunikationszusammenhängen weitergibt. Dies passiert vor allem in persönlichen Gesprächen, die als ,Encounter'-Situationen der Öffentlichkeit (Gerhards/Neidhardt 1990), oder „episodischen Kneipen-, Kaffeehaus- oder Straßenöffentlichkeit[en]" (Habermas 1998, 452) beschrieben wurden und von sehr begrenzter ,Reichweite' sind. Internetkommunikation, insbesondere Soziale Onlinenetzwerke, erweitern die Potenziale für Einzelne sich öffentlich zu äußern, und stellen kommunikative Verknüpfungen zwischen Individuen auf Dauer. Die strukturelle Basis für diese Verknüpfungen sind die technischen Relationen zwischen Nutzeraccounts, die auf Twitter aus Accountperspektive als „Follower" (NutzerInnen, die Tweets eines Accounts „abonniert" haben) und „Followees" (NutzerInnen, deren Tweets ein Account „abonniert" hat) bezeichnet werden.

\subsection{Followernetzwerke}

Die Zusammenstellung der individuellen Followees - der „persönlichen Öffentlichkeit“ der NutzerInnen (Schmidt 2009) - obliegt allein den Individuen, die jeweils entscheiden, welchen der anderen NutzerInnen sie sich zuwenden, indem sie diesen ,folgen. Dabei werden die Mitteilungen eines anderen Accounts zur rückwärts-chronologisch und ständig aktualisierten Liste aller Mitteilungen der Kontakte - dem Newsfeed - hinzugefügt. Wiewohl diesem Folgen im Detail unterschiedliche und facettenreiche Praktiken zugrundeliegen können (Schmidt, 2009, spricht von "Informations-, Beziehungs- und Identitätsmanagement"), können wir davon ausgehen, dass ein wie auch immer geartetes grundsätzliches Interesse an den jeweiligen NutzerInnen und deren Mitteilungen eine entscheidende Rolle bei der Auswahl spielt. Die Struktur der Followernetzwerke von Nutzergruppen aus Makroperspektive spiegelt so die vorherrschenden Interessenskonstellationen innerhalb einer Nutzergruppe wider. Mit der Struktur von Followernetzwerken ist dabei einerseits die Gewichtung einzelner Accounts gemeint, andererseits die Verdichtungen innerhalb des Netzwerks. In diesen finden sich NutzerInnen wieder, die ähnlicheren anderen folgen als der Rest des Netzwerks (Homophilie). Diese Verdichtungen können als Öffentlichkeiten verstanden werden, da sie sich aus der Artikulation von Interessen über die Auswahl von Followees ergeben, und da sie die Beobachtungs- und Handlungspotenziale für die Bezugnahmen der sozialen Akteure langfristig strukturieren.

Dabei ist zu beachten, dass die Beobachtung von Verdichtungen innerhalb bestimmter Nutzergruppen nur sehr begrenzte Ausschnitte der kommunikativen Verbindungen der jeweiligen NutzerInnen repräsentiert, und zwar jeweils jene, die zu anderen Accounts eben dieser Gruppe bestehen. Wie oben angemerkt, ist jede/r NutzerIn Teil verschiedenster Öffentlichkeiten, und dies gilt auch für Kommunikationsstrukturen auf Twitter. Den Blick auf bestimmte Nutzergruppen zu richten bedeutet also, das Interessensumfeld jedes/r Nutzers/in innerhalb eines spezifischen Netzwerkteils zu bestimmen, ihn/sie also einem Segment dieses Ausschnitts zuzuordnen. Dies ist nur dann theoretisch begründbar und praktisch sinnvoll, wenn es sich bei den jeweiligen NutzerInnen tatsächlich um Gruppen mit gemeinsamen Merkmalen handelt - beispielsweise den Beteiligten an einem bestimmten Diskurs.

Eine gute Möglichkeit zur Bestimmung themenzentrierter Öffentlichkeiten bieten Hashtags, da NutzerInnen durch den Gebrauch dieser als Schlagwörter ausgezeichneten Begriffe ihre Beteiligung an einem bestimmten Diskurs artikulieren und ihre Mitteilungen gleichzeitig einem breiteren Publikum zugänglich machen (Bruns/ Burgess 20IIa). Die Bestimmung von Themenöffentlichkeiten mittels Hashtags stößt jedoch überall dort an ihre Grenzen, wo sich NutzerInnen zwar zu geteilten Interessen austauschen, die entsprechenden Mitteilungen aber nicht mit Hashtags markieren. In diesen Fällen können geteilte Interessen gegebenenfalls auch über die gemeinsame Verwendung spezifischer und unverwechselbarer Begriffe bestimmt werden, die einen Themenkomplex möglichst eindeutig repräsentieren. Dabei wird davon ausgegangen, dass alle NutzerInnen, die regelmäßig Mitteilungen zu einem Thema absetzen, diese Begriffe verwenden, und dadurch auf ihr Interesse an dem Themengebiet geschlossen werden kann.

Einschränkend ist anzumerken, dass keineswegs für jedes über Begriffe repräsentierte Thema eine spezifische Öffentlichkeit existiert. Wir können aber davon ausgehen, dass eine hohe Dichte der informationellen Verknüpfungen zwischen solcherlei identifizierten Nut- 
zerInnen ein guter Indikator dafür ist, dass es sich unserer Definition gemäß um Öffentlichkeiten handelt. Die vorliegende Untersuchung basiert auf der Annahme, dass Tweets mit Nennungen österreichischer Parteien durch die terminologische Eindeutigkeit der Bezeichnungen (mit Ausnahme der Grünen, siehe Forschungsdesign) dem Thema „österreichische Innenpolitik“ zugeordnet werden können. Damit nehmen alle NutzerInnen, die einen solchen Tweet absetzen, am innenpolitischen Diskurs des Landes teil und sind so Teil der österreichischen innenpolitischen Öffentlichkeit (auf Twitter).

\subsection{Forschungsfragen}

Wir verstehen Twitter als Kommunikationssystem, in dem Informationen und Meinungen zu mehr oder weniger abgrenzbaren Themengebieten vermittelt werden. Die strukturelle Basis dieser Vermittlung ist ein Netzwerk miteinander verknüpfter Accounts, das die primären themenspezifischen Beobachtungs- und Beteiligungsfelder für die NutzerInnen strukturiert. Gleichzeitig repräsentiert das Accountnetzwerk die aggregierten Interessenskonstellationen innerhalb der an den Themenöffentlichkeiten beteiligten Nutzergruppen. Das Ziel dieser Studie ist, die innere Struktur des Netzwerks zum Thema österreichische Innenpolitik zu identifizieren, wobei wir insbesondere Netzwerkverdichtungen als Teilöffentlichkeiten in den Blick nehmen:

FF1: Wie ist die Öffentlichkeit zu österreichischer Innenpolitik auf Twitter strukturiert, welche Teilöffentlichkeiten lassen sich identifizieren?

FF2: Wie sind diese Teilöffentlichkeiten miteinander verknüpft?

Im Anschluss an diese Strukturanalysen stellen sich unter Einbeziehung netzwerkexogener Kategorien Fragen zur Einordnung der Ergebnisse in den Gesamtzusammenhang politischer Kommunikation in Österreich:

FF3: Wo sind die traditionell wichtigsten Akteure der politischen Öffentlichkeit, PolitikerInnen und Journalist/nnen, in dieser Twitter-Öffentlichkeit verortet?

FF4: Wie ist das Verhältnis dieser Struktur zur österreichischen Parteien- und Medienlandschaft zu bewerten?

\section{Forschungsdesign}

Unser explorativer Forschungsansatz ist methodologisch in den „Digital Methods“ (Rogers, 2009) zu verorten, einer Forschungsperspektive, bei der die computergestützte Analyse der Manifestation von Kommunikationsprozessen in der sozio-technischen Struktur des Internet im Zentrum steht. Dabei werden keine neuen Daten erhoben, sondern es werden bestehende Daten ausgehoben und einer Analyse zugeführt. Twitter ist für solche Studien besonders gut geeignet, da es über eine Programmierschnittstelle („Application Programming Interface", API) verfügt, über die mittels Anfragen umfangreiche Datenbestände abgerufen werden können. So können nicht nur einzelne Tweets ausgehoben werden, sondern auch detaillierte Metainformationen zu den Nutzern und ihren Verbindungen. Für die vorliegende Studie haben wir unterschiedliche Funktionalitäten der API genutzt, um Tweets zu identifizieren, die dem Thema österreichische Innenpolitik zugeschlagen werden können (siehe Abschnitt 4.I.), sowie um an Informationen zu und Verknüpfungen zwischen den Nutzeraccounts zu gelangen, die diese Tweets geschrieben haben (4.2.), um sie anschließend als Netzwerk analysieren zu können (4.3.).

\subsection{Identifikation der Tweets zum Thema}

In den Kalenderwochen 3 bis 3I des Jahres 20I3, also vom I4. Jänner bis zum 4. August, haben wir alle Tweets gesammelt, die den Namen einer österreichischen Parlamentspartei (BZÖ, FPÖ, Grüne, ÖVP, SPÖ, Team Stronach) oder deren jeweiligen Vorsitzenden enthielten. Dieser lange Zeitraum war notwendig, um möglichst alle relevanten NutzerInnen zu identifizieren. Dabei gingen wir davon aus, dass Personen, die sich auf Twitter intensiv über österreichische Innenpolitik austauschen, diese Parteibezeichnungen oder Namen der Vorsitzenden zumindest gelegentlich verwenden. Die Kontrolle der Daten in einer Stichprobe ( $\mathrm{n}=500$ je Datensatz) zeigte, dass sich die Tweets zu unseren Begriffen tatsächlich alle auf österreichische Innenpolitik bezogen. Lediglich bei den Daten zur Partei „Die Grünen“ mussten wir die Daten semi-automatisch nachbearbeiten, da hier eine terminologische Gleichheit zur deutschen Partei besteht.

Die gesamte Datenbank zu Tweets enthielt I85.818 Nennungen von II.776 verschiedenen Accounts in I49.69I verschiedenen Tweets. Tabelle I zeigt die Verteilung von Nennungen im Untersuchungsmaterial. 
Tabelle 1: Anzahl der Nennungen nach Partei und SpitzenkandidatIn

\begin{tabular}{lccccccc}
\hline & ÖVP & FPÖ & SPÖ & Team Stronach & BZÖ & Die Grünen & Gesamt \\
\hline Parteiname & 40.150 & 31.079 & 26.984 & 10.978 & 12.802 & 7.821 & 129.814 \\
SpitzenkandidatIn & 6.697 & 12.107 & 8.844 & 24.434 & 1.286 & 2.636 & 56.004 \\
Gesamt & 46.847 & 43.186 & 35.828 & 35.412 & 14.088 & 10.457 & 185.818 \\
\hline
\end{tabular}

\subsection{Identifikation von Accounts und Verknüpfungen}

Im nächsten Schritt haben wir die im Themenbereich österreichische Innenpolitik aktivsten Nutzeraccounts identifiziert. Dabei haben wir jede/n NutzerIn einbezogen, von dem/der zumindest zehn der in der Grundgesamtheit gesammelten Tweets stammten. Unser Forschungsinteresse ist die Analyse einer themenspezifischen Community, deren Mitglieder sich durch die aktive Beteiligung an dem Thema qualifizieren, bei denen also eine anhaltende mehrfache Beteiligung vorliegt. Die Grenzziehung bei mindestens zehn Tweets ist dabei als forschungspragmatische Entscheidung vor dem Hintergrund technischer Restriktionen einzustufen: Zum einen legt die Twitter-API bestimmte zeitliche Limits für die Abfrage von Nutzerinformationen fest, die die Erhebung größerer Datenmengen massiv beeinträchtigen. Zweitens stoßen die angewandten netzwerkanalytischen Verfahren und Visualisierungstechniken bei besonders dichten Netzwerken schnell an die Grenzen des Machbaren, zumindest mit der uns zum Forschungszeitpunkt zur Verfügung stehenden Rechenleistung. Da sich unsere Fragestellung auf netzwerkglobale Strukturphänomene und nicht auf einzelne Accounts und ihre Verknüpfungen bezieht, halten wir diese Reduktion für vertretbar.

Für die so identifizierten insgesamt I.952 NutzerInnen wurden am 07. August 2013 mit einem leicht modifizierten open-source Python Skript (Russel 20II, 380) über die API Profildaten erhoben. Für I.852 NutzerInnen, also in knapp 95\% der Fälle, war diese Erhebung erfolgreich. Die fehlgeschlagenen Anfragen sind auf Namensänderungen oder gelöschte Accounts zurückzuführen. Anschließend wurden für alle Accounts die Followees abgefragt, wobei all jene Verbindungen, die nicht zu bereits in der Datenbank vorhandenen NutzerInnen führten, entfernt wurden. Auf dieser Basis konnten wir das Netzwerk der Verknüpfungen der I.852 NutzerInnen berechnen und eine Clusteranalyse durchführen.

\subsection{Clusteranalyse}

Öffentlichkeiten als Räumeverdichteter Kommunikation lassen sich mit Methoden der sozialen Netzwerkanalyse definitionsgetreu als Communities bzw. Cluster operationalisieren: Sie sind dadurch beschrieben, dass die Anzahl der Verbindungen innerhalb einer Community proportional höher ist als die Anzahl der Verbindungen zu Knoten, die außerhalb liegen (Newman 2006, 8577). Für die Berechnung von Communities stehen zahlreiche Vorgehensweisen zur Verfügung, wobei die Auswahl eines bestimmten Algorithmus auf Basis der Operationalisierung und der Rechenperformance erfolgt (Leskovec 20IO). Für diese Studie wurde der von Pons und Latapy vorgeschlagene "Walktrap“-Algorithmus auf das Netzwerk der Followerbeziehungen angewandt. Dieser Algorithmus beruht auf der Annahme, dass zufällige gewählte Pfade in einem Netzwerk (,random walks“) mit höherer Wahrscheinlichkeit zwischen Knoten der gleichen Community verlaufen. Daraus ergibt sich ein Maß struktureller Ähnlichkeit, mithilfe dessen der Algorithmus einzelne Knoten zu Communities aggregiert (Pons/Latapy 2005).

Die Auswahl dieses Algorithmus erfolgte aufgrund verschiedener Kriterien. Neben der allgemein gut eingestuften Performance des Algorithmus (Oman/ Labatut 2009, 249) verlangt unser explorativer Zugang nach einer Methode der strukturellen Identifikation ohne vorherige Festlegung auf Anzahl oder Größe der Cluster. Zudem war ein Algorithmus nötig, der auch mit beschränkten Rechenkapazitäten vergleichsweise große und dichte Netzwerke bewältigen kann. Schließlich war die Verfügbarkeit des Algorithmus bzw. die Möglichkeit für seine Implementierung in die von uns genutzte Analysesoftware ${ }^{6}$ mitausschlaggebend für die Auswahl.

Zusätzlich zur Identifikation der Cluster und der Berechnung der lokalen Clusterkoeffizienten selbst haben wir die Verknüpfungen der Cluster untereinander analysiert. Dabei haben wir die gemeinsamen Verbindungen zwischen den Knoten der unterschiedlichen Cluster berechnet.

6 igraph(Csardi/Nepusz 2006) für R. 


\subsection{Quantitative und qualitative Beschreibung der Cluster}

Die Anwendung dieses Algorithmus stellt uns vor eine methodische Herausforderung für eine quantitative Untersuchung: Wir finden Gruppen, die zueinander stärker verknüpft sind als mit anderen Knoten. Diese sind gemäß unserer Kantendefinition als Interessensgemeinschaften zu interpretieren, wobei die geteilten Interessen erst in einem zweiten Interpretationsschritt sichtbar wurden, indem wir in einer qualitativen und quantitativen Analyse der Accountgruppen Schlüsse zur inhaltlichen Ausrichtung der Communities ziehen (vgl. zu diesem Vorgehen auch Jürgens 20I2, I95).

So haben wir in einem induktiven Verfahren an Hand der Beschreibungen der Nutzeraccounts die Gemeinsamkeiten der jeweiligen Communities identifiziert. Bei diesem Verfahren kommt jenen Accounts mehr Relevanz zu, die innerhalb des jeweiligen Clusters zent$\mathrm{ral}^{7}$ sind, weil die Zentralität eines Accounts innerhalb des Clusters als Grad der Übereinstimmung darüber zu verstehen ist, wie interessant dessen Inhalte für die anderen Mitglieder sind. Um weitere Anhaltspunkte zur Interpretation der Cluster zu erhalten, haben wir zudem grundsätzliche Merkmale der Accounts in den Clustern im Durchschnitt berechnet (Followerzahl, Alter der Accounts) sowie die zentralsten Accounts und die in den Tweets der NutzerInnen jeweils verlinkten Domains identifiziert. All diese Daten dienten als Grundlage für die Benennung, Beschreibung und Interpretation der Communities, wie wir sie in Abschnitt 5.I. vornehmen.

\subsection{Kodierung nach Akteursgruppen}

Mit Blick auf Forschungsfrage 3 und parallel zur Clusteranalyse haben zwei KodiererInnen die NutzerInnen auf Basis der Profilinformationen den Akteursgruppen "Politik“, "Journalismus" und „andere“ zugeschlagen, wobei volle Übereinstimmung erreicht wurde. $\mathrm{Zu}$ Politik-Accounts wurden MandatsträgerInnen in Bund, Ländern und Vorfeldorganisationen sowie KandidatInnen zum Nationalrat gerechnet und sie wurden einschließlich einer Parteizuordnung kodiert. Als Journalismus-Accounts wurden all jene Akteure identifiziert, die eine entsprechende Angabe in der Profilinformation getätigt oder ein bestimmtes Medium als Arbeitsplatz genannt hatten.
7 Das hier verwendete Zentralitätsmaß ist der Indegree. Dieser misst die Anzahl der eingehenden Verbindungen eines Knotens und damit in unserem Anwendungsfall die Anzahl der Follower innerhalb unseres Netzwerkes.

\section{Ergebnisse}

Die Ergebnisse der Untersuchung werden nachstehend in Reihenfolge der Forschungsfragen dargestellt. Zuerst werden die identifizierten Communities vorgestellt und inhaltlich beschrieben (Abschnitt 5.I. zu FFI), wobei hier noch einmal darauf hingewiesen werden soll, dass Benennung und Beschreibung der Cluster aus der Aggregatanalyse der gruppierten Nutzerprofile hervorgingen und nicht auf jeden einzelnen Fall zurückgeführt werden können. In einem nächsten Schritt werden diese Communities auf ihr Naheverhältnis innerhalb des Netzwerkes überprüft (5.2. zu FF2). Danach werden die Akteursgruppen der JournalistInnen und politischen MandatsträgerInnen innerhalb des Netzwerkes verortet (5.3. zu FF3). Anschließend folgt eine Interpretation vor dem Hintergrund der österreichischen Parteienlandschaft (6. zu FF4).

\section{1 Öffentlichkeiten / Communities}

Das Gesamtnetzwerk der Followerbeziehungen weist eine Dichte von 0,07I, eine durchschnittliche Pfadlänge von 2,I3 sowie einen globalen Clusterkoeffizient von o,33 auf. Die durchschnittliche Anzahl der Verbindungen beträgt II6,6.

Für das Netzwerk der Followerbeziehungen ergeben sich insgesamt $\mathrm{I} 3$ größere ${ }^{8}$ Communities. Abbildung I zeigt die Cluster im Gesamtnetzwerk. Die Anordnung der Knoten basiert auf dem Algorithmus „Force Atlas 2", der versucht, strukturelle Verdichtungen in visuelle Verdichtungen zu übersetzen (Jacomy et al. 20I4). Deshalb erscheinen die Communities in der Abbildung „regional“ geordnet. In Tabelle 2 finden sich ausgewählte Merkmale der Communities, die zusätzlich zur qualitativen Analyse der Nutzerprofile für die nachfolgende Beschreibung herangezogen wurden.

\section{Medien}

Der größte Cluster (497 Accounts) ist überaus stark an traditionellen Massenmedien orientiert. Unter den zentralsten NutzerInnen finden sich vor allem JournalistInnen sowie NutzerInnen, die primär JournalistInnen folgen. Die NutzerInnen gehören mit durchschnittlich über 33.000 Tweets zu den produktivsten des Netzwerkes, die durchschnittliche Followerzahl ist mit I.I92 vergleichsweise sehr hoch. Da JournalistInnen in Österreich früh damit begonnen haben Twitter zu nutzen (vgl. Abschnitt 2.2.), gehören diese Accounts mit einem durchschnittlichen Alter von 3 Jahren zu den ältesten im Netzwerk. Der zentralste Account dieses Clusters,

8 Es handelt sich dabei um jene Cluster, die zusammen zumindest I\% der NutzerInnen im Ausgangssample ausmachen. 
Abbildung 1: Follower Netzwerk der Accounts der 13 größten Cluster

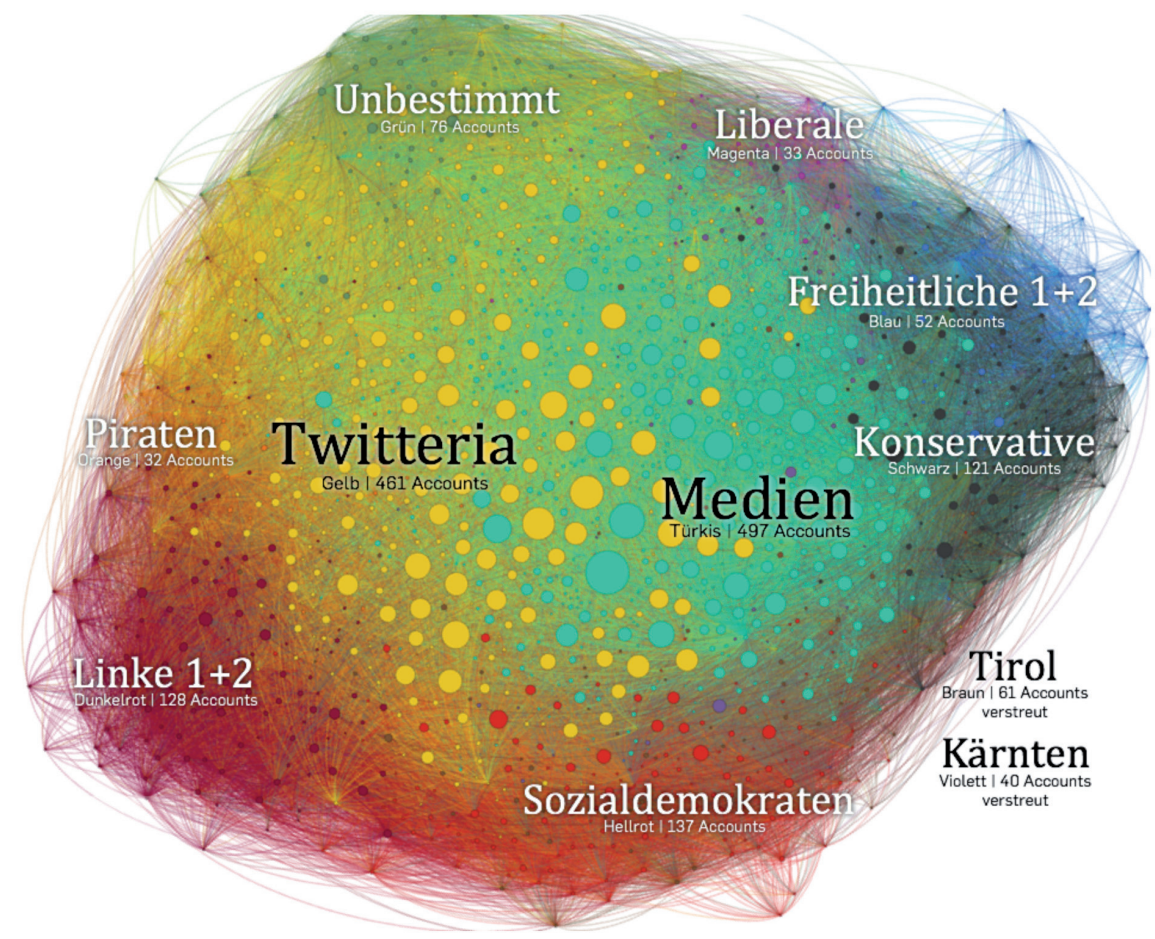

Nodes $=1.638$ (von 1.852), Edges $=191.045$, C.-Algorithmus: Walk-Trap (Steps=2), Visualisierung: Gephi, Force Atlas 2, Node Size= InDegree

der TV-Journalist Armin Wolf, wird von 86,7 Prozent der anderen Accounts des Clusters gefolgt und ist auch gleichzeitig der zentralste des Gesamtnetzwerks. Andere zentrale Accounts sind jener der Onlinetageszeitung derstandard.at sowie Journalist Florian Klenk von der Wiener Stadtzeitung Falter.

\section{„Twitteria“}

Die NutzerInnen in diesem Cluster (46I Accounts) arbeiten vielfach in Berufsfeldern rund um Marketing/ PR und Medientechnologie und zählen zu den „Early Adopters" von Twitter in Österreich. Die Accounts dieses Cluster sind mit 3,7 Jahren die durchschnittlich ältesten des Netzwerkes und verfügen über die höchste durchschnittliche Zahl an Followern (I.265). Der hier gewählte Name „Twitteria“ setzt sich dabei aus „Twitter" und dem Wiener Ausdruck für die gehobene Gesellschaft, „Schickeria“, zusammen. Dieser Begriff wird von den Akteuren selbst häufig zur Bezeichnung der Akteursgruppe genutzt ${ }^{9}$ (vgl. auch Maireder 20I4). Zentrale NutzerInnen, wie der Kommunikationsberater Rudi Fußi, die Journalistin Corinna Milborn und der Politikwissenschafter Hubert Sickinger, waren schon in der Studie zur politischen Twittersphäre im Erhebungszeitraum 20II zentral (Ausserhofer/Maireder 20I3). Zudem

9 http://futurezone.at/netzpolitik/so-zwitschert-oesterreichs-polittwitteria/24.578.740. fällt auf, dass im Twitteria-Cluster zahlreiche PolitikerInnen der Grünen und einige KandidatInnen der NEOS vertreten sind.

\section{Sozialdemokratie}

Der drittgrößte Cluster mit I37 Accounts besteht zum Großteil aus deklarierten Mitgliedern oder Anhängern der SPÖ inklusive zweier Vorfeldorganisationen, dem Verein sozialistischer Studentinnen und Studenten Österreichs (VSStÖ) und der Sozialistischen Jugend (SJ). Der SJ-Vorsitzende Wolfgang Moitzi ist der zentralste Nutzer innerhalb des Clusters (gefolgt von 74,5 Prozent der Accounts) und reiht sich damit noch vor die ehemalige Nationalratsabgeordnete Sonja Ablinger und den offiziellen Account der Bundespartei @spoe_at. Interessant ist, dass der Kärntner SPÖ-Landeshauptmann Peter Kaiser nicht Teil dieses Clusters ist, sondern sich in einem regionalen Kärnten-Cluster wiederfindet (siehe unten).

\section{Konservative}

In diesem Cluster mit I2I Accounts finden sich zu einem großen Teil Funktionäre der Österreichischen Volkspartei und ihrer Vorfeldorganisationen sowie Personen, die sich in ihren Beschreibungen als "ÖVPler" oder "Schwarze" deklarieren. Unter den zentralsten NutzerInnen innerhalb des Clusters finden sich auffällig viele inner- 
Tabelle 2: Überblickstabelle für die 13 größten Cluster

\begin{tabular}{|c|c|c|c|c|c|c|c|}
\hline Benennung & $\begin{array}{l}\text { Anzahl } \\
\text { Accounts }\end{array}$ & $\begin{array}{l}\text { Tweets im } \\
\text { Sample ges. }\end{array}$ & $\begin{array}{l}\emptyset \text { Follower } \\
\text { und St.abw. } \\
\text { [1] }\end{array}$ & $\begin{array}{l}\varnothing \text { Alter der } \\
\text { Accounts \& } \\
\text { St.abw. [2] }\end{array}$ & $\begin{array}{l}\text { Zentralster } \\
\text { Account }\end{array}$ & $\begin{array}{l}\text { \% Follower } \\
\text { Topaccount [3] }\end{array}$ & $\begin{array}{l}\varnothing \text { Lokaler } \\
\text { Clustering- } \\
\text { Koeffizient }\end{array}$ \\
\hline Medien & 497 & 33117 & $\begin{array}{l}1.192 \\
\sigma=4.834\end{array}$ & $\begin{array}{l}3,0 \\
\sigma=1,5\end{array}$ & ArminWolf & 86.7 & $\begin{array}{l}0,51 \\
\sigma=0,14\end{array}$ \\
\hline Twitteria & 461 & 42714 & $\begin{array}{l}1.265 \\
\sigma=2.029\end{array}$ & $\begin{array}{l}3,7 \\
\sigma=1,5\end{array}$ & HubertSickinger & 69.2 & $\begin{array}{l}0,44 \\
\sigma=0,12\end{array}$ \\
\hline Soz.dem. & 137 & 6532 & $\begin{array}{l}353 \\
\sigma=501\end{array}$ & $\begin{array}{l}2,8 \\
\sigma=1,5\end{array}$ & Wmoitzi & 74.5 & $\begin{array}{l}0,52 \\
\sigma=0,11\end{array}$ \\
\hline Kons. & 121 & 7822 & $\begin{array}{l}338 \\
\sigma=410\end{array}$ & $\begin{array}{l}2,9 \\
\sigma=1,4\end{array}$ & Svejk & 85.1 & $\begin{array}{l}0,51 \\
\sigma=0,11\end{array}$ \\
\hline Linke 1 & 95 & 4503 & $\begin{array}{l}530 \\
\sigma=586\end{array}$ & $\begin{array}{l}3,6 \\
\sigma=1,5\end{array}$ & Porrporr & 63.2 & $\begin{array}{l}0,45 \\
\sigma=0,10\end{array}$ \\
\hline Unbest. & 76 & 4726 & $\begin{array}{l}309 \\
\sigma=502\end{array}$ & $\begin{array}{l}2,7 \\
\sigma=1,4\end{array}$ & Tschanschpange & 67.1 & $\begin{array}{l}0,48 \\
\sigma=0,11\end{array}$ \\
\hline Tirol & 61 & 2702 & $\begin{array}{l}365 \\
\sigma=791\end{array}$ & $\begin{array}{l}2,9 \\
\sigma=1,6\end{array}$ & IbkTwit & 83.6 & $\begin{array}{l}0,49 \\
\sigma=0,10\end{array}$ \\
\hline Kärnten & 40 & 1146 & $\begin{array}{l}423 \\
\sigma=1.001\end{array}$ & $\begin{array}{l}3,2 \\
\sigma=1,4\end{array}$ & Kleinezeitung & 72.5 & $\begin{array}{l}0,51 \\
\sigma=0,11\end{array}$ \\
\hline Linke 2 & 33 & 1025 & $\begin{array}{l}518 \\
\sigma=422\end{array}$ & $\begin{array}{l}3,3 \\
\sigma=1,4\end{array}$ & anarchismus_at & 81.8 & $\begin{array}{l}0,51 \\
\sigma=0,10\end{array}$ \\
\hline Liberale & 33 & 1133 & $\begin{array}{l}162 \\
\sigma=167\end{array}$ & $\begin{array}{l}3,0 \\
\sigma=1,4\end{array}$ & Veitdengler & 87.9 & $\begin{array}{l}0,52 \\
\sigma=0,09\end{array}$ \\
\hline Piraten & 32 & 1868 & $\begin{array}{l}282 \\
\sigma=671\end{array}$ & $\begin{array}{l}2,8 \\
\sigma=1,7\end{array}$ & Fisima_ & 78.1 & $\begin{array}{l}0,48 \\
\sigma=0,10\end{array}$ \\
\hline Freih. 1 & 31 & 3517 & $\begin{array}{l}420 \\
\sigma=682\end{array}$ & $\begin{array}{l}2,5 \\
\sigma=1,1\end{array}$ & HCStracheFP & 77.4 & $\begin{array}{l}0,46 \\
\sigma=0,09\end{array}$ \\
\hline Freih. 2 & 21 & 1834 & $\begin{array}{l}126 \\
\sigma=131\end{array}$ & $\begin{array}{l}1,4 \\
\sigma=0,9\end{array}$ & FPOE_TV & 76.2 & $\begin{array}{l}0,57 \\
\sigma=0,11\end{array}$ \\
\hline
\end{tabular}

Anmerkungen: [1] Durschnittliche Gesamt-Followerzahl (nicht nur im Sample!) und Standardabweichung. Die Standardabweichung ist zum Teil deutlich höher als die durchschnittlichen Follower. Dies deutet auf einige wenige NutzerInnen mit besonders hohen Followerzahlen hin; [2] Durchschnittliches Alter der Accounts in Jahren und Standardabweichung; Stichtag: 1.1.2014; [3] Prozent der Accounts im Cluster, die dem Top-Account folgen.

institutionelle Accounts, also solche, die nicht mit dem Namen einer Einzelperson, sondern einer bestimmten Teilorganisation der Partei auftreten. Der zentralste Nutzer - Svejk - ist laut seinem Profil selbst kein Funktionär, sondern Parteimitarbeiter. Aus den Profildaten geht außerdem hervor, dass viele der NutzerInnen aus Niederösterreich stammen oder zumindest dort arbeiten. Darauf deutet auch der Umstand hin, dass gut $7 \%$ aller Links aus diesem Cluster auf die Homepage der Niederösterreichischen Nachrichten (noen.at) verweisen.

\section{Linke 1 und 2}

Jene beiden Cluster, die wir „Linke“ bzw. „Linksaktivismus" nennen, können dem österreichischen Parteienspektrum nicht eindeutig zugeordnet werden. In den Profilbeschreibungen dominieren Begriffe wie „Antifaschismus", „Antirassismus" und „Feminismus“. Die Nut-
zerInnen des Clusters verlinken in ihren Tweets besonders häufig auf stopptdierechten.at. Auch wenn sich einige wenige NutzerInnen als "kommunistisch“ bezeichnen und sehr vereinzelt auch die KPÖ genannt wird, kann der Cluster insgesamt nicht der KPÖ zugeschlagen werden. An dieser Stelle sei jedoch darauf hingewiesen, dass die Suchbegriffe für die Datensammlung nur die Parlamentsparteien enthielten. Es ist möglich, wenn auch nicht wahrscheinlich, dass dadurch weitere KPÖ-Parteiaccounts nicht enthalten sind.

Häufiger als die Nennung dieser Partei ist die Selbstzuordnung der Akteure zur Österrreichischen HochschülerInnenschaft (ÖH) oder zu Studierendenvertretungen und studentischen Basisgruppen. Es fällt auf, dass die Accounts in Linke I zu den ältesten gehören, es ist also auch anzunehmen, dass viele dieser Accounts schon zur Zeit der Proteste an den österreichischen Universitäten im Herbst 2009 aktiv waren, bei denen Twit- 
ter eine wichtige Rolle gespielt hat (vgl. Abschnitt 2.2). Immerhin $37 \%$ aller NutzerInnen dieser Cluster haben ihr Profil 2009 erstellt. Auch wenn die Clusteranalyse zwei Communities identifiziert hat, konnte auf Grund der Nutzerprofile keine eindeutige inhaltliche Differenzierung zwischen den beiden Linksaktivismus Clustern ausgemacht werden, wobei die Verknüpfung zwischen den beiden Clustern außerordentlich stark ist (siehe 5.2). Netzwerkanalytisch sind die beiden Cluster mit der unterschiedlich ausgeprägten Verknüpfung der Accounts mit den zentralen Clustern "Medien“ und "Twitteria“" zu erklären. So scheinen NutzerInnen in "Linke 2 " primär einander zu folgen, während „Linke I"-Accounts auch anderen NutzerInnen im Netzwerk folgen (siehe Tabelle 3).

\section{Unbestimmt}

Der Algorithmus identifizierte auch einen Cluster, dessen inhaltlicher Zusammenhang selbst nach ausführlicher Analyse der Profilinformationen nicht restlos geklärt werden konnte. Viele NutzerInnen verwenden in ihren Beschreibungen Begriffe wie "Literatur" und "Musik". Ob und in welcher Form diese Verweise auf das gemeinsame Thema "Kultur" schließen lassen, ist aber unklar, denn im Gegensatz zu den anderen Communities sind die Indikatoren für die thematische Zuschreibung deutlich schwächer ausgeprägt.
Tirol

Neben politisch zuordenbaren Communities konnten wir zwei Cluster identifizieren, die primär Akteure aus einem bestimmten Bundesland umfassen. Einer dieser beiden Cluster ist "Tirol“ (6I Accounts), wobei die meisten NutzerInnen im Feld "Location“ der Profildaten einen Ort in Tirol oder das Bundesland angeben. Der zentralste Account nennt sich @ Ibktwit und veröffentlicht Nachrichten zur Hauptstadt Innsbruck. Vor diesem Hintergrund überrascht es nicht, dass fast $8 \%$ aller Links, die die NutzerInnen dieses Clusters geteilt haben, auf die Webseite der Tiroler Tageszeitung (tt.com) verwiesen.

\section{Kärnten}

Dieser Cluster setzt sich aus 40 Accounts zusammen, die zum allergrößten Teil Kärnten oder Orte in diesem Bundesland als Standortinformation anführen oder in anderer Weise innerhalb der Profilbeschreibung auf Kärnten Bezug nehmen. Besonders auffällig ist in diesem Fall, dass einige der zentralen Akteure die Kleine Zeitung als Arbeitgeber erwähnen. Dies erklärt, warum kleinezeitung.at der Topaccount des Clusters ist und warum beinahe 30\% der Links aus dieser Nutzergruppe auf die Homepage der Zeitung führen. Auch der Kärntner Landeshauptmann Peter Kaiser ist in diesem Cluster zu finden.

Tabelle 3: Verknüpfungen der zusammengeführten Communities

\begin{tabular}{lcccccccccccccc}
\hline & Medien & Twitteria & Soz.d. & Kons. & Linke1 & Unb. & Tirol & Kärnten & Liberale & Linke2 & Piraten & Freih.1 & Freih.2 \\
\hline Medien & 44,1 & 39,5 & 4,5 & 4,5 & 1,9 & 1,4 & 0,6 & 1 & 0,6 & 0,2 & 0,3 & 1,3 & 0,2 \\
Twitteria & 31,6 & 47,2 & 4,5 & 3,3 & 4,7 & 3,4 & 1,4 & 0,9 & 0,8 & 0,8 & 0,7 & 0,6 & 0 \\
Soz.dem. & 30,9 & 32,3 & 24,9 & 3,2 & 4,6 & 0,5 & 0,7 & 0,8 & 0,2 & 0,8 & 0,2 & 0,6 & 0,1 \\
Kons. & 36,1 & 25,9 & 3,8 & 27,9 & 0,7 & 0,6 & 1,2 & 1,1 & 0,7 & 0,1 & 0,2 & 1,5 & 0,2 \\
Linke1 & 19,5 & 44,3 & 6,7 & 0,8 & 17,4 & 0,9 & 1,3 & 1 & 0,2 & 7,3 & 0,5 & 0,2 \\
Unb. & 25,8 & 45,5 & 1,8 & 1,6 & 2,2 & 19,4 & 1,4 & 0,5 & 0,3 & 0,5 & 0,5 & 0,5 & 0 \\
Tirol & 26,2 & 33,9 & 4,9 & 5,5 & 3,1 & 2 & 21 & 1 & 0,4 & 0,5 & 0,3 & 0,9 & 0,1 \\
Kärnten & 35,7 & 34,5 & 3,3 & 3,6 & 3,1 & 0,7 & 1,2 & 16 & 0,2 & 0,1 & 0,5 & 1,1 \\
Liberale & 31 & 37,4 & 2,2 & 3,8 & 1,1 & 0,9 & 0,2 & 0,3 & 21,4 & 0,3 & 0,9 & 0,4 & 0 \\
Linke2 & 7,4 & 27,5 & 5,3 & 0,1 & 33,4 & 1 & 0,6 & 0 & 0,1 & 24,3 & 0,2 & 0,1 & 0 \\
Piraten & 24,7 & 42,7 & 2,7 & 1,8 & 4,1 & 1,2 & 0,7 & 1,2 & 1,1 & 0,2 & 19,1 & 0,4 & 0,1 \\
Freih.1 & 44 & 24,4 & 2,8 & 5,4 & 0,7 & 1,4 & 0,9 & 0,8 & 0,1 & 0,1 & 0,1 & 12,2 \\
Freih.2 & 29,8 & 5,8 & 1,2 & 2,9 & 0,5 & 0,2 & 0,2 & 0 & 0 & 0,2 & 0 & 36,6 & 22,8 \\
\hline
\end{tabular}




\section{Liberale}

Der Cluster setzt sich hauptsächlich aus NutzerInnen zusammen, die sich als liberal beschreiben oder ihre $\mathrm{Zu}$ gehörigkeit zu einer politischen Organisation ausdrücken, die als liberal verstanden werden kann. Mehr als zwei Drittel der 33 NutzerInnen nennen in ihrer Profilbeschreibung NEOS, JuLis, das LiF oder deklarieren sich politisch als liberal. Obwohl sich für diesen Cluster sehr einfach eine politische Tendenz identifizieren lässt, fällt auf, dass zentrale Akteure der NEOS wie Matthias Strolz und Niko Alm nicht diesem Cluster zugeordnet sind, sondern sich in den zentralen Communities "Twitteria“ und "Medien“ finden.

\section{Piraten}

Die zentralsten der 32 NutzerInnen in diesem Cluster beschreiben sich als Mitglieder der Piratenpartei oder einfach als „Piraten“. Wenig überraschend spielt in den Profilinformationen auch Technik und insbesondere Informationstechnologie eine wichtige Rolle. Der Account von Philip Pacanda, Pirat im Gemeinderat Graz, ist der zentralste Knoten innerhalb der Gruppe.

\section{Freiheitliche 1 und 2}

Der Algorithmus identifizierte zwei verschiedene Cluster, deren NutzerInnen mehrheitlich an der Freiheitlichen Partei Österreichs orientiert sind. Im Großteil der Beschreibungen der Accounts finden sich Verweise auf die Partei oder die Vorfeldorganisation Ring Freiheitlicher Jugend (RFJ), häufig werden auch Positionen innerhalb der Partei bzw. des RFJ angegeben. Unterschiede zwischen den beiden Clustern sind kaum zu identifizieren, es fällt lediglich auf, dass die politischen Accounts in „Freiheitliche I“ (3I Accounts) eher in der Bundespolitik tätig sind, während die professionellen politischen Profile in "Freiheitliche 2" (2I Accounts) tendenziell in der Regional- oder Landespolitik verortet werden können. Insgesamt handelt es sich um junge Accounts (zwischen I,5 und 2,5 Jahre alt) und sehr kleine Cluster. Der Twitterauftritt von Heinz Christian Strache ist zwar der zentralste innerhalb des Clusters "Freiheitliche I", es handelt sich dabei jedoch um einen Account, der nur automatisiert Meldungen aus Straches Facebook-Profil weiterleitet. Auffällig ist zudem, dass die NutzerInnen dieses Clusters die einzigen sind, die häufig auf kronenzeitung.at verlinken ( $9,07 \%$ aller Tweets mit Hyperlinks).

\subsection{Verknüpfung der Öffentlichkeiten}

Tabelle 2 schlüsselt die Verbindungen der aggregierten Cluster zueinander auf. Die Zahlen spiegeln dabei den Prozentanteil der kumulierten ausgehenden Follower- beziehungen eines Clusters auf den jeweiligen anderen wider. Die zweite Zelle der ersten Spalte bedeutet beispielsweise, dass 31,6\% aller ausgehenden Verbindungen der NutzerInnen aus dem Cluster "Twitteria“ auf NutzerInnen des Clusters "Medien“ gerichtet sind.

Die Cluster „Twitteria“ und "Medien“ sind das Zentrum des Netzwerkes. Insgesamt sind fast zwei Drittel aller Kontakte der verbleibenden Cluster sind auf diese beiden Nutzergruppen gerichtet, wobei bedacht werden sollte, dass die beiden Cluster gleichzeitig die größten des Netzwerkes darstellen. Hierbei fällt auf, dass sich alle anderen Cluster auch auf Basis ihrer Beziehung zu diesen beiden Hauptclustern unterscheiden lassen. Während die Cluster „Konservative" und "Freiheitliche“ stärker dem Mediencluster folgen, sind alle anderen Nutzergruppen stärker auf die „Twitteria“ ausgerichtet. Letzteres gilt in besonderem Maße für die beiden Subcluster "Freiheitliche 2 " und "Linke 2 ". „Freiheitliche 2 " folgen nur zu 5,8\% dem Cluster "Twitteria" und „Linke 2“ nur zu 7,4\% dem Cluster Medien. Der unterschiedliche Verknüpfungsgrad mit den zentralen Clustern ist auch ein Grund für die netzwerktopologische Zersplitterung der Linken und Freiheitlichen in jeweils zwei Communities.

Wirft man einen genaueren Blick auf jene Cluster, deren Profilanalyse politische Übereinstimmungen ergeben haben, zeigt sich folgendes Bild: Wenig überraschend bestehen die stärksten Beziehungen zwischen jenen Clustern, die auch inhaltlich anhand der Profilbeschreibungen nur minimal voneinander unterschieden werden konnten, namentlich "Linke I" und "Linke $2^{\text {" }}$ bzw. „Freiheitliche I" und „Freiheitliche 2 ". Zudem fällt auf, dass die NutzerInnen des Clusters „Linke I“ relativ stark (6.7\%) dem Cluster „Sozialdemokratie“ folgen und diese Beziehung ebenso erwidert wird (4.6\%). Letzteres trifft auf die Followerbeziehungen zwischen „Freiheitlichen“ und „Konservativen" nur in sehr beschränktem Maße zu. Während zumindest 5.4\% der Followerbeziehungen von Seiten „Freiheitliche I" auf NutzerInnen im konservativen Cluster verweisen, sind insgesamt nur I.7\% der Followerbeziehungen aus diesem Cluster auf "Freiheitliche I" und „Freiheitliche 2 " gerichtet. Asymmetrische Kanten dieser Art finden wir auch zwischen den Clustern "Liberale“ und „Konservative“ bzw. zwischen "Piraten" und „Linke I“. Wenig überraschend zeigt sich zudem, dass die Verbindungen zwischen den Clustern „Linke“ und „Freiheitliche“" zu den Schwächsten des Netzwerkes gehören.

\subsection{Politik und Journalismus im Netzwerk}

Auf Basis der Kodierung nach Akteursgruppen (siehe 4.5) können wir des Weiteren Aussagen über die Position von Journalismus- und Politik-Accounts machen: Wie bereits aus den Clusterbeschreibungen deutlich wurde, 
finden sich Journalismus-Accounts vornehmlich in dem eigenen Cluster "Medien“ (zu ca. 60\%) und „Twitteria“ (zu knapp 30\%) wieder. Nur 12 von 219 JournalistInnen beziehungsweise Medienaccounts sind in politisch zuordenbaren Clustern verortet. Es handelt sich dabei um fünf solcher Accounts im Cluster „Linke I", vier im Cluster "Konservative“ und drei im Cluster „Sozialdemokratie“.

In Abbildung I wurde bereits deutlich, dass die Cluster "Medien“ und „Twitteria“ das Zentrum des Netzwerkes bilden. Dieses Ergebnis lässt sich auch auf jene Accounts erweitern, die wir anhand ihrer Twitter-Profilbeschreibungen als journalistische Accounts identifizieren konnten. Der durchschnittliche Indegree dieser Accounts sagt aus, dass im Schnitt 268 NutzerInnen aus dem in Abbildung I gezeigten Netzwerk JournalismusAccounts folgen. Für PolitikerInnen beträgt dieser Wert 156 und für alle verbleibenden NutzerInnen liegt er bei nur 86. Journalistische Accounts haben also eine zentrale Position im Netzwerk und sind für die Vermittlung von Fakten und Meinungen von besonderer Bedeutung.

Politik-Accounts finden sich beinahe ausnahmslos an der Peripherie des Netzwerkes. Zudem (und wenig überraschend) lässt sich feststellen, dass diese Accounts in hohem Maße in jenen Clustern zu finden sind, die wir schon in einem ersten Schritt politischen Strömungen zuordnen konnten. So befinden sich beispielsweise alle Accounts der Freiheitlichen Partei in einem der beiden dieser Partei zugeordneten Cluster. Ähnliches gilt für die beiden Großparteien, so finden sich 3I von 32 Accounts der ÖVP im Cluster „Konservative“ und 32 von 37 SPÖAccounts im Cluster „Sozialdemokratie“. Accounts der Partei NEOS stellen in diesem Zusammenhang einen Sonderfall dar.
Auch für die beiden Kleinparteien Piraten und das Wahlbündnis NEOS/LiF/JuLis lassen sich Cluster identifizieren, wobei auffällt, dass nur 6 von II dezidierten NEOS Accounts innerhalb des Clusters verortet sind und zentrale Akteure der NEOS wie Spitzenkandidat Matthias Strolz, JuLis-Vorsitzender Niki Scherak und Kandidat Niko Alm der „Twitteria“ zuzuordnen sind. Dies bedeutet, dass ihnen NutzerInnen des „Twitteria“ Clusters verhältnismäßig stärker folgen, und sie so in ein weitläufigeres und zentraleres Kommunikationsnetzwerk eingebettet sind. Dies gilt auch und ganz besonders für die Grünen, für deren FunktionärInnen und MandatarInnen sich kein eigener Cluster ausmachen lässt. Zwei Drittel der Accounts finden sich im Cluster "Twitteria“ (z.B. Michel Reimon, Marco Schreuder, Harald Walser, Sigi Maurer, u.a.), I6\% in "Tirol“ und II\% in "Medien“. Nur zwei der 32 grünen Accounts sind in einem politisch zuordenbaren Cluster zu finden, nämlich „Linke I“. Die zentralen Akteure des BZÖ (Stefan Petzner, Heimo Lepuschitz, Josef Bucher) finden sich im Cluster "Medien“. Das Team Stronach ist mit seinem Parteiaccount ebenfalls in diesem Cluster vertreten.

\section{Interpretation und Diskussion}

Das auffälligste Ergebnis der Netzwerkanalyse sind die Parallelen zwischen den Clustern und der Struktur der österreichischen Parteienlandschaft. So sind zumindest drei Communities eindeutig österreichischen Parlamentsparteien zuzuordnen: Sozialdemokraten (SPÖ), Konservative (ÖVP) und Freiheitliche (FPÖ), auch wenn letztere durch die unterschiedlich ausgeprägten Followerbeziehungen $\mathrm{zu}$ anderen Teilen des Netzwerks (v.a. dem Cluster „Medien“) eher zersplittert sind. Für

Abbildung 2: Zugehörigkeit der Politik-Accounts zu den jeweiligen Clustern

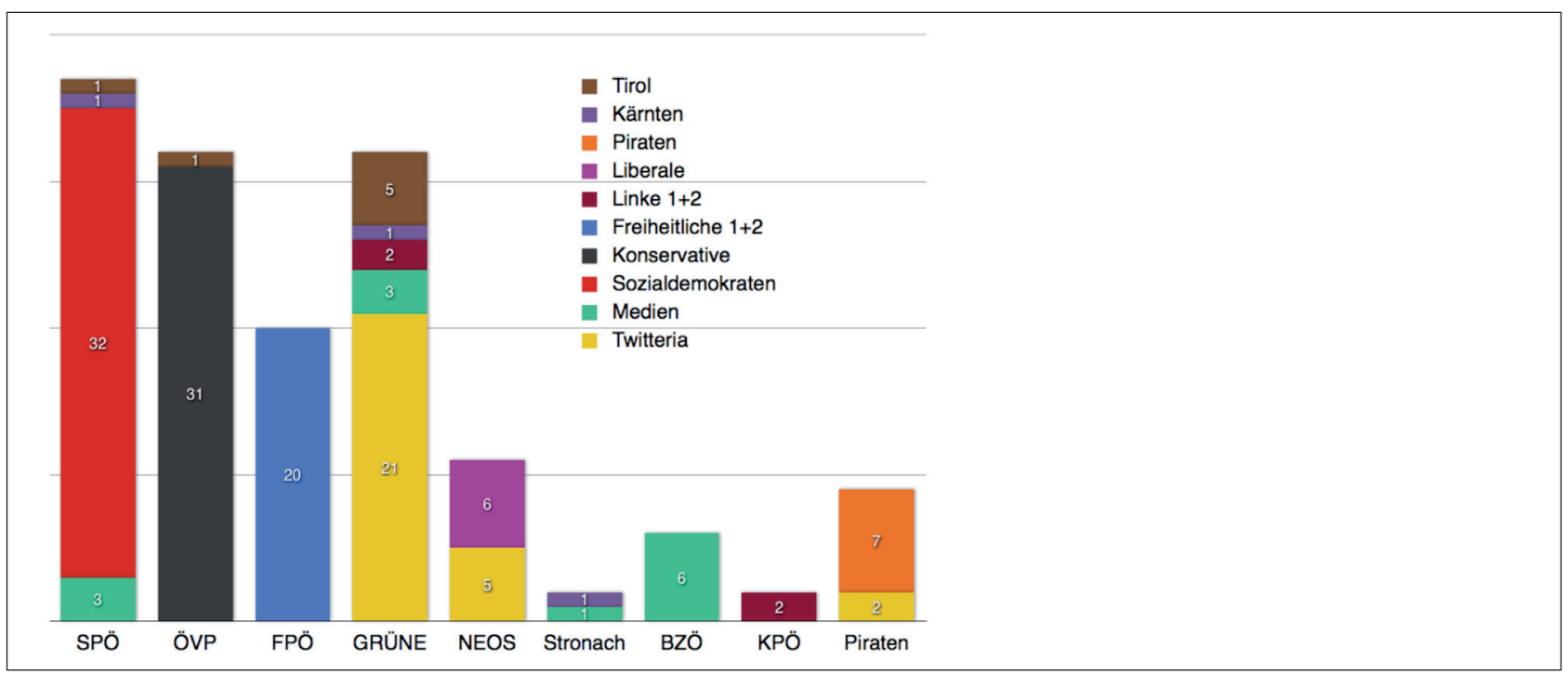


Akteure in diesen Clustern erscheint Twitter so auch als Medium zur (öffentlichen) Kommunikation unter Parteifreunden, wahrscheinlich zur gegenseitigen $\mathrm{Ab}-$ stimmung über Positionen und Aktivitäten der Partei. Auch die beiden Cluster "Linke I" und "Linke 2" bilden eine solche politisch-ideologisch einordenbare Teilöffentlichkeit, die jedoch keiner Partei zugeordnet werden kann. Viele Accounts dieser Community wurden auch schon in der Studie aus 20II über ihre Interaktionen als gut vernetzte Gruppe identifiziert (Ausserhofer/ Maireder 20I3) und dort mit dem Terminus "politischer Aktivismus" bezeichnet. Hier scheinen sich politisch aktive BürgerInnen ein verschiedene linke Gruppierungen übergreifendes Kommunikationsnetzwerk geschaffen $\mathrm{zu}$ haben, über das nicht nur Informationen ausgetauscht werden, sondern in dem auch politische Aktionen und Demonstrationen koordiniert werden. Letzteres legen die Ergebnisse von Maireder/Ausserhofer (2013) zur Twitter-Nutzung während der Proteste gegen den Ball rechter Burschenschafter (WKR-Ball) nahe.

Die politisch eindeutig zuordenbaren Cluster sind allesamt am Rande des Gesamtnetzwerks positioniert. Dies deutet darauf hin, dass die Tweets der NutzerInnen eine im Durchschnitt vergleichsweise begrenzte Gruppe von aktiven NutzerInnen erreichen, wie dies auch die durchschnittlichen Followerzahlen nahelegen. Für GrünpolitikerInnen sowie für Teile der NEOS gilt dies nicht im selben Maße: Da diese Accounts in zentrale und politisch nicht eindeutig verortbare Teilöffentlichkeiten integriert sind, erreichen ihre Mitteilungen eine im Durchschnitt deutlich breitere Öffentlichkeit. Dies kann für politische Kommunikationsprozesse strategisch vorteilhaft sein. Warum es diesen Accounts gelingt zentrale Positionen einzunehmen, kann anhand rein struktureller Ergebnisse nicht beantwortet werden. Vorsichtig lassen sich jedoch zwei Vermutungen formulieren: Einige PolitikerInnen und institutionelle Accounts der Österreichischen Grünen gehören zu den „Early Adopters“ des Microbloggingdienstes Twitter in Österreich. Weil Followerbeziehungen relativ stabile Netzwerktopologien ausbilden, kann davon ausgegangen werden, dass es für lange aktive NutzerInnen vergleichsweise einfach ist, Positionen im Zentrum zu besetzen und diese auch zu halten. Letzteres gilt in besonderem Maße für grüne PolitikerInnen (wie zum Beispiel Sigi Maurer) und Persönlichkeiten der NEOS (wie Niko Alm). Weiters darf vermutet werden, dass sich die sogenannte „Twitteria“ auch inhaltlich am ehesten auf politische Inhalte der Grünen und der NEOS verständigen kann, selbst wenn der Cluster nicht von diesen dominiert wird.

In der Perspektive auf die Positionierungen der Akteursgruppen zueinander zeigen sich Konstellationen, die vor dem Hintergrund der politisch-ideologischen Einordnung der NutzerInnen in höchstem Maße nachvollziehbar erscheinen. An der Peripherie des Netz- werks scheint sich von Piraten und Linken über Sozialdemokraten hin zu Konservativen und Freiheitlichen das politische Links-Rechts-Spektrum zu entfalten. Ideologisch nähere Positionen spiegeln sich so in der jeweils näheren Positionierung der Akteursgruppen, mit Linken und Freiheitlichen an den gegenüberliegenden Enden des Graphen. Auch die grünen PolitikerInnen innerhalb der „Twitteria“ sind deutlich näher an Linken und Sozialdemokraten positioniert als viele andere Akteure dieses Clusters. Die Positionierung der Accounts in den beiden regionalen Clustern "Kärnten“ und "Tirol“ ist im Gesamtkontext schwierig zu verorten. Dies legt die Interpretation nahe, dass sich in den beiden Bundesland-Communities NutzerInnen unterschiedlicher politischer Einstellungen zusammenfinden, die untereinander verhältnismäßig stärker verknüpft sind als mit politisch zuordenbaren Accounts.

Mit Blick auf das Zentrum des Netzwerks ist die herausragende Rolle des Journalismus zu betonen, der auf Twitter (und der Netzöffentlichkeit insgesamt) zwar seine Alleinstellung als "Gatekeeper" von der Politik zum Publikum verloren hat, aber nichtsdestotrotz eine zentrale Vermittlungsrolle in den innenpolitischen Diskursen einnimmt. Dabei sind weniger Medienunternehmen als einzelne JournalistInnen aktiv, die sich als (politisch interessierte) Individuen und Politik-ExperInnen präsentieren. Sie verweisen in den Profilbeschreibungen zwar meist auf ihre Arbeitgeber, betonen jedoch gleichzeitig, dass ihre Tweets persönliche Meinung widerspiegeln. Neben dem „Journalismus“ ließ sich mit der sogenannten „Twitteria“ jedoch eine zweite, ebenso zentrale, Akteursgruppe identifizieren, die aus den klassischen Rollenkonzepten im politischen System herausfällt. Aus den Nutzerbeschreibungen lässt sich herauslesen, dass viele der NutzerInnen in medien- und politiknahen Bereichen beschäftigt sind, und/oder in ihrer Freizeit gesellschaftspolitisch aktiv sind, ohne jedoch parteipolitische Präferenzen zu kommunizieren. Da die Accounts dieser politisch interessierten BürgerInnen der Zivilgesellschaft ein fixer Bestandteil des Informationsrepertoires zahlreicher politischer MandatsträgerInnen und JournalistInnen sind, prägen sie den Diskurs rund um österreichische Innenpolitik nicht unwesentlich mit.

\section{Conclusio}

Twitter ist ein durch Accountnetzwerke strukturiertes, transparentes und beteiligungsoffenes Kommunikationssystem, in dem sich soziale Akteure (Individuen und Kollektive) durch das Absetzen von Kurzmitteilungen zu vielfältigen Themen austauschen. Eines dieser Themen ist österreichische Innenpolitik. In der vorliegenden Studie wurden jene NutzerInnen identifiziert, die sich an Diskussionen zu diesem Thema in 28 Untersu- 
chungswochen im ersten Halbjahr 2013 beteiligten. Anschließend wurden die Verknüpfungen zwischen diesen Accounts erhoben und als Netzwerk analysiert. Im Zentrum stand dabei die Frage nach der Struktur dieses Netzwerkausschnitts, die einerseits Rückschlüsse auf die Relevanz einzelner Akteure und andererseits auf die Herausbildung von Clustern und deren Positionierung zueinander ermöglicht. Diese Verdichtungen im Netzwerk haben wir schließlich als (Teil-)öffentlichkeiten verstanden, da die Accountverknüpfungen die Beobachtungs- und Beteiligungsmöglichkeiten der NutzerInnen grundlegend strukturieren.

Die österreichischen innenpolitischen Kommunikationsprozesse auf Twitter sind von I3 solchen Öffentlichkeiten geprägt, von denen acht politisch-ideologisch eindeutig kategorisierbar sind. Die Beobachtung von und Beteiligung an politischen Kommunikationsprozessen erscheint für diese NutzerInnen primär auf politisch ähnlich positionierte Accounts gerichtet, und kann so von den NutzerInnen als Kommunikation unter Gleichgesinnten erlebt werden - auch wenn die Kommunikationsprozesse selbst öffentlich und beteiligungsoffen sind. Dies kann als Indikator für die Partikularisierung von Öffentlichkeit in neuen Medien verstanden werden, wie sie vielfach diskutiert wurde (vgl. zusammenfassend Farell 2012). Dagegen spricht die starke Verknüpfung zwischen den einzelnen Communities: Die NutzerInnen folgen zwar stärker Gleichgesinnten, beschränken sich in ihrem Followee-Repertoire aber bei weitem nicht auf die eigene Community; ganz im Gegensatz zu Studien wie jener von Adamic \& Galance (2005), die auf Basis von Verlinkungen zwischen US-Blogs ausgesprochen ausgeprägte republikanische und demokratische Communities identifizierten.

Die Parallelen zwischen den politischen Clustern und der österreichischen Parteienlandschaft sind vielfältig, sodass von einer spezifischen Form der Repräsentation des österreichischen politischen Systems auf Twitter gesprochen werden kann. Da Twitter als Medium der Kommunikation unter Akteuren des politischen Systems (im weitesten Sinne) zusehends an Bedeutung gewinnt, ist diese Struktur auch für die Aushandlungsprozesse politischer Deutung von Relevanz. Dabei ist die außerparlamentarische Opposition (Linke, Piraten und zum Zeitpunkt der Erhebung auch Liberale) im Verhältnis zu den etablierten Parteien (SPÖ, ÖVP, FPÖ) stark überrepräsentiert. Soziale Medien ermöglichen diesen Gruppen verstärkt Aufmerksamkeit zu erlangen, wobei die primären Adressaten wohl JournalistInnen sind, von denen wohl gehofft wird, dass sie diese Aufmerksamkeit gegebenenfalls in einen massenmedialen Kontext übertragen. Journalismus scheint eine tragende Rolle zu spielen, da die Verknüpfungen zwischen den unterschiedlichen politischen Teilöffentlichkeiten auch auf Twitter vielfach über den Cluster „Medien“ als Zentrum des Netzwerks laufen. Daneben konnte sich jedoch auch ein alternatives zweites Zentrum etablieren, das von zivilgesellschaftlichen Akteuren getragen wird.

So liefert diese Studie vor dem Hintergrund der Frage nach den Chancen neuer Medien für das Aufbrechen traditioneller politischer Strukturen und der Inklusion neuer politischer Akteure durchaus gemischte Ergebnisse: Es zeigt sich einerseits, dass politisch-ideologische Grenzziehungen auch auf Twitter relevant sind und dass der Journalismus eine zentrale Vermittlungsrolle in politischen Kommunikationsprozessen einnimmt. Andererseits nehmen alternative Akteure sowohl innerhalb der politischen Teilöffentlichkeiten als auch im Gesamtnetzwerk relevante Positionen ein und die Grenzziehungen zwischen den politischen Teilöffentlichkeiten sind zwar bestimmbar aber zuweilen außerordentlich durchlässig. Für PolitikerInnen, die sich in diesen fluiden Öffentlichkeiten zentral positionieren, entstehen neue Chancen für die Kommunikation politischer Standpunkte. Zumindest NEOS und die Grünen scheinen diese Potenziale im vergangenen Wahlkampf für sich genutzt zu haben.

Neben konkreten Erkenntnissen zur Struktur der österreichischen politischen Twittersphäre zeigt unsere Studie Potenziale von Netzwerkanalysen der Accounts sozialer Onlinenetzwerke für die politische Kommunikationsforschung auf. Die sozio-technische Struktur der Netzwerke liegt dabei (öffentlichen) Kommunikationsprozessen ebenso zu Grunde, wie sie in und durch diese Prozesse geschaffen wird. Dadurch können Strukturanalysen sozialer Onlinenetzwerke nicht nur Erkenntnisse zur konkreten Nutzung dieser neuen Medientechnologien liefern, sondern vor dem Hintergrund ihrer zunehmenden Bedeutung für politische Kommunikation auch zur Segmentierung der Öffentlichkeit an sich.

\section{Literatur}

Anderson, Christopher W. (2010). Journalistic Networks and the Diffusion of Local News: The Brief, Happy News Life of the "Francisville Four," in: Political Communication, Vol. 27, 289-309.

Anduiza, Eva/Marta Cantijoch/Aina Gallego (2009). Political Participation and the Internet, in: Information, Communication \& Society, Vol. I2(6), 860-878.

Ausserhofer, Julian/Maireder, Axel (2013). National Politics on Twitter, in: Information, Communication \& Society, Vol. I6(3), 29I-3I4.

Benkler, Yochai (2007). The Wealth of Networks, New Haven, London.

Bruns, Axel/Jean Burgess (2011a). The use of Twitter hashtags in the formation of ad hoc publics, in: 6th European Consortium for Political Research General Conference, University of Iceland, Reykjavik. 
Bruns, Axel/Jean Burgess (20IIb). \#Ausvotes: How twitter covered the 2010 Australian federal election, in: Communication, Politics \& Culture, Vol. 44(2), 37-56.

Bruns, Axel/Tim Highfield (2013). Political Networks on Twitter, in: Information, Communication \& Society, Vol. I6(5), 667-69I.

Davis, Areon (2009). New media and fat democracy: the paradox of online participation, in: New Media \& Society, Vol. I2(5), 745-76I.

Edelmann, Noella/Peter Parycek/Judith Schoßböck (20II). The unibrennt movement: a successful case of mobilising lurkers in a public sphere, in: International Journal of Electronic Governance, Vol. 4(I), 43-68.

Emirbayer, Mustafa/Mimi Sheller (1999). Publics in history, in: Theory and Society, Vol. 28(I), I43-I97.

Farrell, Henry (2012). The Consequences of the Internet for Politics, in: Annual Review of Political Science, Vol. I5(I), 35-52.

Gerhards, Jürgen/Friedhelm Neidhardt (1990). Strukturen und Funktionen moderner Öffentlichkeit: Fragestellungen und Ansätze, in: Veröffentlichungsreihe der Abteilung Öffentlichkeit und Soziale Bewegungen des Forschungsschwerpunkts Sozialer Wandel, Institutionen und Vermittlungsprozesse des Wissenschaftszentrums Berlin für Sozialforschung, Vol. FS III, 90-IOI.

Golbeck, Jennifer/Justin M. Grimes/Anthony Rogers (2010). Twitter use by the U.S. Congress, in: Journal of the American Society for Information Science and Technology, Vol. 6I(8), I6I2-I62I.

Grant, Will J./Brenda Moon/Janie Busby Grant (20I0). Digital Dialogue? Australian Politicians' use of the Social Network Tool Twitter, in: Australian Journal of Political Science, Vol. 45(4), 579-604.

Gruning, James E./Todd T. Hunt (1984). Managing Public Relations, New York.

Habermas, Jürgen (1998). Faktizität und Geltung: Beiträge zur Diskurstheorie des Rechts und des demokratischen Rechtsstaats, Frankfurt am Main.

Henn, Philipp/Marco Dohle/Gerhard Vowe (2013). „Politische Kommunikation": Kern und Rand des Begriffsverständnisses in der Fachgemeinschaft, in: Publizistik, Vol. 58(4), 367-387.

Hepp, Andreas/Michael Brüggemann/Katharina Kleinenvon Königslöw/Swantje Lingenberg/Johanna Möller (2012). Ein Beschreibungs- und Erklärungsansatz: Transnationale Öffentlichkeit und politische Diskurskulturen in Europa, in: Politische Diskurskulturen in Europa, Wiesbaden, $2 \mathrm{I}-47$.

Herwig, Jana/Max Kossatz/Viola Mark (2010). \#unibrennt im Internet. Beobachtungen zu einer sich ändernden Protestqualität, in: Stefan Heissenberger/Viola Mark/ Susanne Schramm/Peter Sniesko/Rahel Sophia Süß (Hg.): Uni Brennt, Wien-Berlin, 2IO-22I.
Jacomy, Mathieu/Tommaso Venturini/Sébastien Heymann/Mathieu Bastian (20I4). ForceAtlas2, a Continuous Graph Layout Algorithm for Handy Network Visualization Designed for the Gephi Software, in: PLoS ONE, Vol. 9(6), e98679. doi:IO.I37I/journal. pone.oog8679.

Jürgens, Pascal (2012). Communities of Communication: Making Sense of the "Social" in Social Media, in: Journal of Technology in Human Services, Vol. 30(34), I86-203.

Jürgens, Pascal/Andreas Jungherr (20II). Wahlkampf vom Sofa aus: Twitter im Bundestagswahlkampf 2009, in: Eva Johanna Schweitzer/Steffen Albrecht (Hg.): Das Internet im Wahlkampf, Wiesbaden, 20I-225.

Kooti, Farshad/Haeryun Yang/Meeyoung Cha/Krishna P. Gummadi/Winter A. Mason (2010). The Emergence of Conventions in Online Social Networks, in: Proceedings of the Sixth International AAAI Conference on Weblogs and Social Media, I94-20I.

Larsson, Anders Olof/Hallvard Moe (20II). Studying political microblogging: Twitter users in the 20Io Swedish election campaign, in: New Media \& Society, Vol. I4(5), 729-747.

Leskovec, Jure/Kevin J. Lang/Michael Mahoney (2010). „Empirical comparison of algorithms for network community detection." In Proceedings of the I9th international conference on World wide web, 63I-640. Raleigh, ACM.

Maireder, Axel (20I0). Twitter in Österreich: Strukturen, Formen \& Themen österreichischer Tweets. Forschungsbericht, Universität Wien. Internet: http:// homepage.univie.ac.at/axel.maireder/php/wordpress/wp-content/TwitterinOesterreich.pdf $\quad(\mathrm{Zu}-$ griff: I.II.2OI4).

Maireder, Axel (2014). Ein Tweet: Zur Struktur von Netzöffentlichkeit am Beispiel Twitter, in: Heike Ortner/Daniel Pfurtscheller/Michaela Rizzolli/Andreas Wiesinger (Hg.): Datenflut und Informationskanäle, Innsbruck, 55-70.

Maireder Axel/Ausserhofer Julian (2014). Political Discourses on Twitter: Networking Topics, Object and People, in: Katrin Weller/Axel Bruns/Jean Burgess/Merja Mahrt/Cornelius Puschmann (Hg.). Twitter and Society, New York, NY 305-318.

Maireder, Axel/Schlögl, Stephan (20I4). 24 hours of an \#outcry: The networked publics of a socio-political debate, in: European Journal of Communication, Vol. 29(6), 687-702.

Maireder, Axel/Schwarzenegger, Christian (2012). A Movement of Connected Individuals, in: Information, Communication \& Society, Vol. I5(2), I7I-I95.

Neumayer, Christina/Judith Schoßböck, (20II). Political Lurkers? Young people in Austria and their political life worlds online, in: Peter Parycek/Manuel J. Kripp/ 
Noella Edelmann (Hg.): CeDemII - Conference for EDemocracy and Open Government, I3I-I43.

Newman, Mark E. (2006). Modularity and community structure in networks, in: Proceedings of the National Academy of Sciences, Vol. IO3(23), 8577-8582.

Pons, Pascal/Matthieu Latapy (2005). Computing communities in large networks using random walks, in: Computer and Information Sciences-ISCIS, Vol. 3733, 284-293.

Rogers, Richard (2009). The End of the Virtual. Digital Methods, Amsterdam.

Russell, Matthew A. (201I). Mining the Social Web: Analyzing Data from Facebook, Twitter, LinkedIn, and Other Social Media Sites, Beijing.

Schmidt, Jan (2009). Das neue Netz: Merkmale, Praktiken und Folgen des Web 2.0, Konstanz.

\section{Autoren}

Stephan Schlögl war wissenschaftlicher Projektmitarbeiter (Prae-Doc) am Institut für Publizistik- und Kommunikationswissenschaft der Universität Wien und ist seit Februar 2015 wissenschaftlicher Mitarbeiter am Global Social Media Intelligence Center der GfK.

Axel Maireder war Projektleiter des Forschungsprojektes TANEP am Institut für Publizistik und Kommunikationswissenschaft der Universität Wien, und ist seit Jänner 2015 wissenschaftlicher Leiter des Global Social Media Intelligence Center der GfK. 